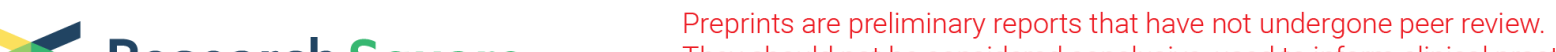 Research Square They should not be considered conclusive, used to inform clinical practice, or referenced by the media as validated information.
}

\section{Development of a Prediction Score for In-Hospital Mortality in COVID-19 Patients with Acute Kidney Injury: A Machine Learning Approach}

Daniela Ponce ( $\nabla$ daniela.ponce@unesp.br)

Universidade Estadual Paulista Júlio de Mesquita Filho https://orcid.org/0000-0002-6178-6938

Luis Gustavo Modelli de Andrade

Universidade Estadual Paulista Julio de Mesquita Filho - Campus de Botucatu

Rolando Claure-Del Granado

Universidad Mayor de San Simon

Alejandro Ferrero Fuentes

Universidad de la República Uruguay: Universidad de la Republica Uruguay

Raul Lombardi

Universidad de la República Uruguay: Universidad de la Republica Uruguay

\section{Research}

Keywords: COVID-19, acute kidney injury, risk factors, machine learning

Posted Date: April 26th, 2021

DOI: https://doi.org/10.21203/rs.3.rs-426545/v1

License: (c) (i) This work is licensed under a Creative Commons Attribution 4.0 International License.

Read Full License

Version of Record: A version of this preprint was published at Scientific Reports on December 1st, 2021.

See the published version at https://doi.org/10.1038/s41598-021-03894-5. 


\section{Abstract}

Introduction: Acute kidney injury (AKI) is frequently associated to COVID-19, and is considered an indicator of severity of disease and is thus associated with increased mortality risk".

Objective: The aim of the study was to develop and validate a prognostic score at hospital admission for predicting in-hospital mortality in COVID-19 patients with AKI (AKI-COV score).

Design: Cross-sectional multicenter prospective cohort study.

Setting: The Latin America AKI COVID-19 Registry has been conducted in 57 cities in 12 countries from Latin America. Model training was performed on a cohort of patients admitted from May 1 to December $31,2020$.

Participants: Eight hundred and seventy COVID-19 patients with AKI defined according KDIGO serum creatinine criteria were included between 01 May to 31 December 2020.

Material and Methods: We evaluated four categories of predictor variables available at the time of AKI diagnosis: (1) demographic data; (2) comorbidities and condition at admission; (3) laboratory exams at admission; (4) characteristics and causes of AKI. We used a machine learning approach to fit models in the training set using 10 -fold-cross validation. Predictors with more than $30 \%$ missing were removed. We select the best model and confirm the accuracy in a validation cohort using the area under the receiver operating characteristic curve (AUC-ROC).

Main Outcome Measured: In-hospital mortality.

Results: There were 544 (62.5\%) in-hospital deaths. Increasing age, mechanical ventilation, use of vasopressors, leukocytes number[RC1] transaminases levels, hypertension, severe condition at admission, AKI ethiology, and need kidney replacement therapies (KRT) were associated with increased risk of death. Longer time from symptoms to hospitalization or to AKI diagnosis, and higher urine output were associated with reduced risk of death. The coefficients of the best model (Elastic Net) were used to build the predictive ImAgeS score. The final model has an AUC-ROC of $0.823[95 \% \mathrm{Cl} 0.761-0.885]$ in the validation cohort.

Conclusion: We developed a predictive model using only demographic data, comorbidities, hospital admission condition, laboratory variables and causes of AKI that shows good accuracy and is easily applicable. The use of AKI-COV score may assist health-care workers in identifying hospitalized COVID-19 patients with AKI that may require more intensive monitoring and can be used for resource allocation.

\section{What Is Already Known On This Topic?}

- The association between AKI in COVID-19 patients and higher mortality is already well established and scoring systems can be useful for fast and effective assessment of mortality risk in AKI patients 
or in COVID-19 patients in the intensive care unit.

- There are few data on factors associated with higher mortality in AKI-COVID 19 patients and no available scores for predicting in-hospital mortality in COVID-19 patients with AKI.

- Derivation and validation studies in low- and middle-income countries, including Latin America, are scarce

\section{What this study adds?}

- AKI-COV Score employs well defined variables, routinely assessed upon hospital presentation: age, mechanical ventilation, use of vasopressor, leukocytes number, time from COVID-19 symptoms to hospitalization or to AKI, severe condition at admission, transaminases levels, AKI etiology, diuresis and need KRT.

- We development a predictive score (AKI-COV) that could be used to AKI associated with COVID-19 risk stratification in hospitalized patients.

\section{Implications of all the available evidence}

- The AKI-COV score may be very useful in a real-world setting, to provide healthcare practitioners the decision support that is needed to help them better identify and prioritize the care of patients who have the higher risk of death. Its development and validation followed strict methodological criteria.

\section{Introduction}

The coronavirus disease 2019 (COVID-19) caused by the severe acute respiratory syndrome coronavirus 2 (SARS-CoV-2) has rapidly spread globally. Currently, America has been the epicenter of COVID-19 pandemic for the past few months, and Brazil has the third worldwide in total number of COVID-19 cases and second number of deaths. The impact of COVID-19 has been devastating on the Latin America, with all regions and all states being affected ${ }^{1,2}$. As of 1 st March, 2021, there are over 20 million confirmed cases and 650,000 deaths, and these figures are probably underestimated ${ }^{3}$.

The respiratory system is the primary target of the virus, but it is increasingly recognized that affects other organs, including the kidneys. Previous studies showed that acute kidney injury (AKI) and kidney abnormalities such as proteinuria and haematuria might be common among patients with COVID-19 ${ }^{4-6}$. Several mechanisms appear to be involved in AKI pathophysiology, including direct injury, cytokine storms, angiotensin-II pathway activation, complement disorders, hypercoagulation states, and microangiopathy interacting known risk factors for $\mathrm{AKI}^{7-8}$.

AKI is associated with increased morbidity and mortality, especially when is severe and associated with other underlying diseases [9]. The estimated incidence of AKI among hospitalized COVID-19 patients varies between studies, ranging from $0.5 \%$ to as high as $60 \%[10,11]$. Two of the largest studies exemplify this wide variation: Guan et al. ${ }^{10}$ reported an AKI incidence of only $0.5 \%$ in an analysis of 1,099 
hospitalized patients across China, while an analysis from New York performed by Hirsch et al reported an AKI incidence of $36.6 \%$ among 5,449 hospitalized COVID-19 patients ${ }^{12}$. A Brazilian study was published recently and showed overall AKI incidence of $50 \%$. Among ICU patients, AKI occurred in $77.3 \%$ and the mortality was $65.4 \%{ }^{13}$.

Many studies showed that COVID-19 patients who developed AKI had increased mortality risk ${ }^{9-14}$. A recent study of 5,449 individuals admitted to hospitals across New York, found that $35 \%$ of patients who developed AKI died, with an adjusted odds ratio (OR) of $9.6^{12}$.

It is important to provide an accurate estimation of mortality in COVID-19 patients with AKI, as well as to explore the differences in these estimations. This approach will improve treatment strategies and facilitate healthcare planning. Considering there lack of data from Latin America, a cross sectional study was performed. Risk factors for death in AKI hospitalized COVID- 19 patients were reported. In addition, a new score for predicting the mortality in COVID-9 patients with AKI was developed.

Predictive scores have been developed to assist risk stratification in AKI or COVID 19 patients ${ }^{15-18}$. Additionally, the predictive models could be particularly useful in supporting the process of decision making of which patient needs urgent assessment or not.

To date, there is no predictive score for assessing the risk of death in COVI-19 patients with AKI. The aim of the present study was to develop and validate a prognostic model for AKI associated with COVID 19 patients using the data of the Latin America COVID-AKI Registry ${ }^{19}$.

\section{Patients And Methods}

This study is part of the Latin America COVID-AKI Registy, an observational, prospective, longitudinal, multinational registry that included COVID-19 patients with AKI in Latin America ${ }^{19}$. An open invitation to participate in the Registry was made through the webpage of the Latin American Society of Nephrology and Hypertension (SLANH), the National Societies of Nephrology in the region and by personal email sent to members of RedIRA (an educational tool of SLANH). Participation in the Registry was voluntary, without any incentive or economic benefit for patients or investigators.

Bioethical considerations: the Institutional Review Board of the Clínica Los Olivos, Cochabamba, Bolivia (contact Dr. Esdenka Vega, administracion@clinicalosolivos.com) approved the study. The informed consent was considered not mandatory by the reference IRB given the observational characteristic of the study. Protocol and forms are available on the study's website (https://slanh.net/registrolatinoamericano-ira-covid-19/). Confidentiality of information was appropriately protected by deidentification of data. No personal data of patients was included in the form.

Inclusion criteria were adult patients with COVID-19 infection confirmed by RT-PCR of nasopharyngeal swabs and acute kidney injury (AKI) from 01 May to 31 December 2020. Exclusion criteria were patients with CKD stage 5, on dialysis or transplanted. Data were obtained from the clinical record of patients and 
were entered online by the participants in a Surveymonkey ${ }^{\circledR}$ platform specifically designed for this purpose (https://es.surveymonkey.com/r/L6PVMGQ).

The form has six sections that included: 1) country and city of residence, demographic data; 2 ) comorbidities and condition at admission; 3) laboratory at admission; 4) characteristics and causes of AKI; 5) ICU admission, mechanical ventilation (MV) and in-hospital complications; 6) condition at discharge.

SLANH provided financial support for the design and maintenance of the platform during the recruitment period, as well as for the publishing fees. The funder had no role in study design, data collection and analysis, decision to publish, or preparation of the manuscript. Competing interests: The authors have declared that no competing interests.

\subsection{Definitions}

AKI was identified according to KDIGO definition when occurred an increase in serum creatinine (SCr) level ${ }^{3} 0.3 \mathrm{mg} / \mathrm{dl}$ within 48 hours or by $50 \%$ within 7 days. AKI was considered as community-acquired (CA-AKI), when it was present at admission or it developed within 48 hours of hospital admission and as hospital-acquired (HA-AKI) when AKI developed after $48 \mathrm{~h}$ hospital stay ${ }^{20}$.

Condition on admission to the hospital was classified in three categories: mild, if the patient was admitted to a conventional ward without need for oxygen therapy; moderate, if patient needs oxygen therapy; and severe, when admission in the intensive care unit (ICU) was needed.

The study followed the guidelines of the transparent reporting of a multivariable prediction model for individual prognosis or diagnosis (TRIPOD) ${ }^{21}$.

\subsection{Predictors variables}

We evaluated the predictors available at the time of COVID-19 diagnosis related to four categories: (1) demographic data; (2) comorbidities and condition at admission; (3) laboratory exams at admission; (4) characteristics and causes of AKI (supplementary material). The comorbidities evaluated were diabetes, obesity, hypertension, lung disease, neoplasia, liver disease, autoimmune disease, cardiovascular disease, and smoking. We also evaluated the time from COVID-19 symptoms to hospitalization and from hospitalization to AKI.

\subsection{Outcome}

The outcome evaluated was in-hospital death. Use of medications to treat COVID-19, admission in intensive care unit, and indication for mechanical ventilation were performed at each center according to their own local practices.

\subsection{Statistics analysis}




\section{Exploratory Data Analysis}

All variables of interest were compared between patients who survived with those who died in-hospital. This comparison was performed by the $\mathrm{X} 2$ test for categorical variables and by the Mann-Whitney test for continuous variables.

\subsubsection{Predictive Model}

For the predictive model, the categorical variables were transformed into dummy variables. We removed the variables with more than $30 \%$ missing values and imputed the others using nearest neighbors. Once the nearest neighbors are determined, the mode is used to predictor nominal variables and the mean is used for numeric data.

The continuous variables were normalized dividing theirs values by means (center) and standard deviation (scale). We transformed the continuous variable using Box-Cox transformation. Variables with zero or near-zero variance were removed from the model.

\subsubsection{Feature selection}

We used a random forest algorithm to select the most important predictors. We calculate the variable importance measure (VIM) using feature selection with the Boruta algorithm. The method performs a topdown search for relevant features by comparing original attributes' importance with importance achievable at random, estimated using their permuted copies, and progressively eliminating irrelevant features to stabilize that test. We removed the features considering not important by Boruta algorithm and highly correlated variables (above 0.9) (supplementary material).

\subsubsection{Model Training}

We split the data into derivation (training) and validation (test) data sets. To create the datasets, we used a random split stratified by the target into training ( $80 \%)$ and test $(20 \%)$. In the training data (derivation cohort), 10-fold-cross validation was used to select the hyperparameters of the models and to reduce the bias. We fitted gradient boosting decision trees (xgBoost), random forest, and an Elastic Net to develop the candidate equations. Finally, the best hyperparameters were selected using machine learning approaches by 10 -fold-cross validation in train set aim to maximize the area under the receiver operating characteristic curve ${ }^{22-23}$.

\subsubsection{Assessment of Accuracy}

The accuracy of the derivation cohort model was tested on the data of the validation cohort. We used the area under the receiver operating characteristic curve (AUC-ROC) to discriminate ability of the models in train and test set. The $95 \%$ confidence interval $(\mathrm{Cl})$ of AUC-ROC was estimated by bootstrap resampling $(2,000$ samples) to reduce overfit bias. We evaluate the calibration of models with Brier and a calibration plot (supplementary). 


\subsubsection{Score Fit and Model visualization}

The model with higher AUC-ROC in the validation cohort and better calibration values was used to build AKI score. We used Shapley Additive Explanations (SHAP) to visualize and explain the importance of the predictors.

The software R version 4.0.2 and the packages tidymodels and DALEx were used to create and visualize the models. The R package "glmnet" statistical software (R Foundation) was used to perform the Elastic Net regression ${ }^{24}$.

\section{Results}

\subsection{Demographic data, the COVID-19 infection presentation and difference between survivor and non-survivor AKI patients}

Between May and December 2020, 967 COVID-19 patients with AKI were identified in 57 centers and 12 countries from Latin America that were part of the SLANH COVID-19 - AKI Registry.

Median age was 63 (54-74) years; 595 were male (68.4\%), 759 (87.2\%) had one or more comorbidities being hypertension, diabetes and obesity prevalent among others (Table 1). Time between diagnosis of COVID-19 and hospital admission was $2(0-4)$ days and condition at hospital admission was mild in 121 (14.0\%); moderate in $384(44.2 \%)$ and severe in $363(41.8 \%)$. Of note, about half of patients had a serum creatinine ( $\mathrm{sCr}$ ) at admission within normal values. 
Table 1

Demographic and clinical characteristics of COVID-19 patients with Acute Kidney Injury at hospital admission

\begin{tabular}{|c|c|c|c|c|}
\hline \multirow[t]{2}{*}{ Variable } & General & Non-survival & Survival & \multirow[t]{2}{*}{ p } \\
\hline & $N=870$ & $N=544$ & $N=326$ & \\
\hline Male sex (\%) & $595(69)$ & $383(71)$ & $212(65)$ & 0.10 \\
\hline Age & $632 \pm 14.8$ & $65.1 \pm 13.8$ & $59.2 \pm 16.1$ & 0.0001 \\
\hline \multicolumn{5}{|l|}{ Comorbidities (\%) } \\
\hline Cardiovascular disease & $135(15.5)$ & $94(17.3)$ & $41(12.6)$ & 0.038 \\
\hline Obesity & $278(31.9)$ & $199(36.6)$ & $79(24.2)$ & 0.0001 \\
\hline Condition at admission $\mathrm{n}(\%)$ & & & & 0.0001 \\
\hline Mild & $121(13.9)$ & $36(6.6)$ & $85(26.2)$ & \\
\hline Moderate & $384(44.1)$ & $237(43.6)$ & $147(45.2)$ & \\
\hline Severe & $363(41.7)$ & $270(49.7)$ & $93(28.6)$ & \\
\hline \multicolumn{5}{|c|}{ Laboratory exams at hospital admission } \\
\hline White blood cell count $\left(\mathrm{mm}^{3}\right)$ & $11807 \pm 5518$ & $12607 \pm 6578$ & $\begin{array}{l}10439 \pm \\
2585\end{array}$ & 0.0001 \\
\hline Lymphocytes $/ \mathrm{mm}^{3}$ & $862 \pm 258$ & $850 \pm 201$ & $871 \pm 287$ & 0.02 \\
\hline Ferritine $(\mathrm{ng} / \mathrm{ml})$ & $1285 \pm 1021$ & $1435 \pm 1126$ & $1055 \pm 1022$ & 0.0001 \\
\hline CK (U/L) & $\begin{array}{l}411(289- \\
534)\end{array}$ & $\begin{array}{l}744(576- \\
1433)\end{array}$ & $\begin{array}{l}414(227- \\
602)\end{array}$ & 0.004 \\
\hline D-Dimer (ng/mL) & $1,132 \pm 310$ & $1,298 \pm 337$ & $1,030 \pm 202$ & 0.5 \\
\hline $\begin{array}{l}\text { Time from COVID symptoms to } \\
\text { hospitalization (days) }\end{array}$ & $4.0(1.0-6.0)$ & $2(0.0-3.0)$ & $5.0(1.0-6.0)$ & 0.18 \\
\hline Hospital-acquired AKI n (\%) & $547(62.8)$ & $399(74.0)$ & $148(48.2)$ & 0.0001 \\
\hline \multicolumn{5}{|l|}{ Cause of AKI n (\%) } \\
\hline Hypovolemia & $311(35.7)$ & $156(28.7)$ & $155(47.59$ & 0.0001 \\
\hline MODS SARS-CoV2 & $515(59.2)$ & $376(69.1)$ & $139(42.6)$ & 0.0001 \\
\hline MODS sepsis & $254(29.2)$ & $207(38.1)$ & $47(14.4)$ & 0.0001 \\
\hline Nephrotoxic drugs & $182(20.9)$ & $99(18.2)$ & $83(25.5)$ & 0.007 \\
\hline
\end{tabular}

CK: creatinophosphokinase; AKI: acute kidney injury; ICU: intensive care unit; MODS: multiple organs disfunction syndrome; Scr: serum creatinine 


\begin{tabular}{|c|c|c|c|c|}
\hline Variable & $\begin{array}{l}\text { General } \\
\mathrm{N}=\mathbf{8 7 0}\end{array}$ & $\begin{array}{l}\text { Non-survival } \\
\mathrm{N}=544\end{array}$ & $\begin{array}{l}\text { Survival } \\
N=326\end{array}$ & $\mathbf{p}$ \\
\hline Oliguric AKI (\%) & $336(38.6)$ & $259(48.3)$ & $77(25.0)$ & 0.0001 \\
\hline Scr peak mg/dL & $\begin{array}{l}3.88(2.5- \\
5.1)\end{array}$ & $\begin{array}{l}4.30(2.9- \\
6.3)\end{array}$ & $\begin{array}{l}3.17(2.1- \\
4.1)\end{array}$ & 0.0001 \\
\hline Kidney replacement therapy (\%) & $402(46.2)$ & $315(58.0)$ & $87(27.0)$ & 0.0001 \\
\hline Non-recovery of renal function & $533(61.3)$ & $462(88.5)$ & $71(23.7)$ & 0.0001 \\
\hline $\begin{array}{l}\text { Duration of kidney replacement therapy } \\
\text { (days) }\end{array}$ & $\begin{array}{l}12.4(6.89- \\
16.90)\end{array}$ & $\begin{array}{l}8.19(4.98- \\
10.12)\end{array}$ & $\begin{array}{l}16.5(11.7- \\
21.20\end{array}$ & 0.0001 \\
\hline Admission to ICU (\%) & $622(71.5)$ & $484(88.9)$ & $138(42.3)$ & 0.0001 \\
\hline Mechanical ventilation (\%) & $628(72.2)$ & $492(90.6)$ & $136(42.1)$ & 0.0001 \\
\hline Use of vasopressors (\%) & $527(60)$ & $432(82)$ & $95(29)$ & 0.0001 \\
\hline Minimum $\mathrm{PaO}_{2} / \mathrm{FiO}_{2}$ & $159 \pm 66$ & $140 \pm 46$ & $190 \pm 82$ & 0.0001 \\
\hline New onset of proteinuria & $163(18.7)$ & $106(32.8)$ & $57(24.1)$ & 0.015 \\
\hline New onset of hematuria & 133(15.3) & $101(30.9)$ & $32(13.7)$ & 0.0001 \\
\hline Last available Scr mg/dL & $\begin{array}{l}3.18(1.54- \\
3.83)\end{array}$ & $\begin{array}{l}3.74(1.74- \\
4.02)\end{array}$ & $\begin{array}{l}2.17(1.31- \\
2.90)\end{array}$ & 0.0001 \\
\hline \multicolumn{5}{|l|}{ Complications (\%) } \\
\hline Sepsis & $439(50.4)$ & $360(67.2)$ & $79(25.1)$ & 0.0001 \\
\hline Infection & $76(8.73)$ & $22(4.3)$ & $54(17.4)$ & 0.0001 \\
\hline other complications & $161(18.5)$ & $51(9.9)$ & $110(34.9)$ & 0.0001 \\
\hline Hospital stay (days) & $\begin{array}{l}19.6(13.8- \\
23.9)\end{array}$ & $\begin{array}{l}16.2(12.6- \\
18.5)\end{array}$ & $\begin{array}{l}21.6(15.5- \\
26.1)\end{array}$ & 0.0001 \\
\hline $\begin{array}{l}\text { Time from COVID symptoms to AKI } \\
\text { (days) }\end{array}$ & $5.0(1.0-8.0)$ & $4(1.0-6.0)$ & $7.0(0.0-3.5)$ & 0.18 \\
\hline
\end{tabular}

AKI was hospital-acquired in 547 (62.9\%). Time between diagnosis of COVID-19 and the onset of AKI was 3 (1-7) days. Multiple Organs Disfunction Syndrome (MODS) attributables to SARS-CoV-2 infection was the main cause of AKI (35.7\%). As expected, in many patients AKI was linked to more than one etiology. In the majority of cases AKI was non-oliguric (59.9\%). The most patients had AKI-KDIGO 3 (59.7\%), followed by AKI-KDIGO 1 (25.8\%). Kidney replacement therapy (KRT) was performed in 402 patients (46.2\%). With this regard, it was expressly requested if some patient needing KRT didn't receive it for any reason being identified 43 people in this condition (4.9\%). Reasons were not recorded but presumably 
were due to resource shortages. The most common procedure was intermittent hemodialysis (IHD) (69.1\%), followed by prolonged intermittent renal replacement therapy (PIRRT) (32.8\%), continuous renal replacement therapy (CRRT) (15.6\%) and peritoneal dialysis (PD) (2.7\%). Patients were treated for SARSCoV-2 infection with steroids in $73.9 \%$; chloroquine-hydroxychloroquine in $12.5 \%$; ivermectine in $6.8 \%$; tocilizumab in $1.5 \%$; remdesivir and linipovir-ritonavir in $0.6 \%$. It should be highlighted that in a large proportion of patients the option "other treatment" was selected in $46.9 \%$ with no further specification. Most of the patients had complications during the hospitalization prevailing sepsis in $50.4 \%$ of cases followed by infection without sepsis (8.7\%) and deep venous thrombosis (5.4\%).

All-cause in-hospital mortality was $62.5 \%$ (544 out of 870 patients). Most of the deaths occurred in the ICU (88.9\%). Table 1 shows the variables associated to mortality in the univariate analysis.

\subsection{Development of model prediction risk for COVID-19 associated mortality}

The patients were grouped randomly in two cohorts: the derivation cohort or train set $(n=697,80 \%)$ and the internal validation cohort or test set $(n=173,20 \%)$ as shown in Fig. 1.

We had a total of 44 predictors and remove 7 by higher missing values. We also removed 17 uninformative and 2 colinear predictors (supplementary). Then, we fit predictive models using the candidate predictors ( $\mathrm{n}=18$ predictors).

We fitted several candidate models with 10 -fold cross-validation and analyzed the performance of these models throughout the area under the curve of receiver operating characteristic curves in the derivation cohort. The AUC were 0.894(0.82-0.93), 0.886(0.85-0.95), and 0.877 (0.83-0.93) for Random forest, XgBoost and Elastic Net, respectively. In a second step, the performance of these models was tested in the internal validation cohort. The AUC-ROC was 0.831 (0.76-0.89), $0.823(0.75-0.88)$, and 0.821 (0.750.88) for Random Forest, XgBoost and Elastic Net (Table 2).

To choose the most useful model, we additionally plot AUC-ROC values in the Fig. 2, and a confusion matrix of 28-day mortality in the derivative cohort, shown in Fig. 3 . The performance of the models were similar but the Elastic Net had better calibration values in validation cohort (attachment).

\subsection{Making A Score-based Prediction}

The results of Elastic Net showed that the mechanical ventilation, higher age, vasopressors, higher leukocyte number and aspartate aminotransferase (AST) values at hospital admission, severe/moderate condition at admission, hypertension, hospital acquired AKI, AKI etiology related to sepsis or COVID, higher creatinine levels during hospitalization and indication of KRT were related to worse outcome. The higher urine output, the longer time from COVID symptoms to hospitalization and to AKI diagnosis, the use of nephrotoxic drugs, dehydration and AKI of nephrotoxic or ischemic etiology were related to a better outcome (Fig. 4). The coefficients of Elastic Net model were used to build the AKI-COV score. 


\subsection{Practical Application}

We are showing examples of predictions of four different hypothetical patients (Table 3 ). Patient 1 and 2 had same CA-AKI and mild condition at admission and patient 3 and 4 had HA-AKI and moderate/severe condition at admission. There was need KRT in patients 2 to 4 and mechanical ventilation and vasopresors in patient 4 . In these scenarios, the probability of death was progressive range from $0.5-$ $93 \%$. For better demonstration, the contribution and importance of each predictor are visualized in a SHAP plot, shown in the Fig. 5. Finally, we develop a web app to estimate individual probability for point of care decision, which is available at: https://covidmodels.shinyapps.io/covid_aki_app/

Table 02

Performance metrics (AUC-ROC) of COVID-19 mortality models in derivation cohort and validation cohorts

\begin{tabular}{|lll|}
\hline Model & AUC-ROC & \\
\cline { 2 - 3 } & Derivation cohort $(\mathbf{n}=697)$ & Internal Validation cohort $(\mathbf{n = 1 7 3})$ \\
\hline Random Forest & $0.894[0.82-0.93]$ & $0.831[0.76-0.89]$ \\
\hline XgBoost & $0.886[0.85-0.96]$ & $0.823[0.75-0.88]$ \\
\hline Elastic Net & $0.877[0.83-0.97]$ & $0.821[0.75-0.88]$ \\
\hline [95\% Confidence Interval] based on 2000 bootstrap resample \\
\hline
\end{tabular}


Table 3

COVID-19 mortality prediction (AKI-COV) in four hypothetical AKI patient

\begin{tabular}{|c|c|c|c|c|}
\hline & Patient 1 & Patient 2 & Patient 3 & Patient 4 \\
\hline \multicolumn{5}{|l|}{ DEMOGRAPHY } \\
\hline Age (yeas) & 40 & 50 & 60 & 60 \\
\hline Sex male & Yes & Yes & Yes & Ye \\
\hline Hypertension & No & No & No & No \\
\hline \multicolumn{5}{|l|}{ Time from COVID symptoms to: } \\
\hline - hospital admission (days) & 5 & 10 & 10 & 10 \\
\hline - AKI (days) & 10 & 12 & 12 & 12 \\
\hline Acquired AKI in & Community & Community & Hospital & Hospital \\
\hline Condition at admission & Mild & Mild & Moderate & Severe \\
\hline Dehydratation & Yes & No & No & No \\
\hline Use of Nefrotoxic Drugs & Yes & No & No & No \\
\hline AKI etiology & Other & COVID & Sepsis & COVID \\
\hline Diuresis & Normal & Oliguria & Oliguria & Oliguria \\
\hline \multicolumn{5}{|l|}{ Laboratory } \\
\hline WBC & 10000 & 12000 & 15000 & 18000 \\
\hline AST & 40 & 50 & 60 & 60 \\
\hline Creatinine & 2 & 2.5 & 3 & 2.5 \\
\hline Indication of Renal Replacement & No & Yes & Yes & Yes \\
\hline Mechanical Ventilation & No & No & No & Yes \\
\hline Use of Vasopressors & No & No & No & Yes \\
\hline \multicolumn{5}{|l|}{ PREDICTIONS } \\
\hline Probability 28 days Death & $0.5 \%$ & $12.7 \%$ & $40.8 \%$ & $93 \%$ \\
\hline
\end{tabular}

\section{Discussion}

Previous studies have used machine learning to develop early COVID-19 prognostic models for a specifc severe outcome with overall good performance ${ }^{25-27}$, frequently reaching over 0.90 AUROC ${ }^{28}$. However, 
in the best our knowledge, this is the first multicenter study that developed a model to predict in-hospital mortality COVID-19 patients with AKI and that was performed in Latin America, the new epicenter of pandemic.

In this model, we used predictors easily available on initial diagnosis and at hospital admission including demographics data, time of symptoms to hospitalization and AKI, laboratory exams and AKI characteristics. The model achieved a higher discriminative capacity to detect immediate risks of negative prognosis and can also contribute to the early start of preventive measures and new interventions, and thereby increase AKI COVID - 19 patient survival ${ }^{29,30}$.

The final model fitted was a combination of the least absolute shrinkage and selection operator (LASSO) with the Ridge regression. It was a generalized linear model via penalized maximum likelihood that performs regularization and variable selection ${ }^{18}$. The regularization path is computed for the LASSO penalty at a grid of values for the regularization parameter lambda ${ }^{18}$. The proportion of LASSO and Ridge as well as the amount of regularization was selected by 10 -fold cross-validation. We also confirmed the predictions in an internal validation cohort not used to fit the model.

For mortality of AKI COVID - 19 patients, variable importance analysis identifed that age, mechanical ventilation, vasopressors, higher white blood cell and ALT values at hospital admission, severe/moderate condition at admission, hypertension, hospital acquired AKI, AKI etiology related to sepsis or COVID, and indication of KRT were related to worse outcome. The higher urine output, the shorter time from symptom to hospitalization and to AKI diagnosis, the use of nephrotoxic drugs, the dehydration and nephrotoxic or ischemic AKI were related to a better outcome.

While the age of the patient is widely an important predictor for most negative health outcomes, leukocytosis has been increasingly included among the main inflammatory biomarkers for the prognosis of cardiovascular ${ }^{30}$ and respiratory diseases ${ }^{31}$. Leukocytosis and high levels of CRP have been also previously associated with individual severity of SARS-CoV-2 ${ }^{32,33}$. One of the main causes of death in COVID-19 patients is the unregulated immune response, with an uncontrolled production and secretion of cytokines. Aging is associated with a wellknown decline in the adaptive and innate immunity, which plays a major role in the increased susceptibility of infections ${ }^{34}$. Age-related immune imbalance is also related to an increased severity in pro-inflammatory response and increased cytokine production, what is believed to increase patient vulnerability to the unregulated inflammatory response in COVID-19 ${ }^{35}$.

Interestingly, hypertension, mechanical ventilation and use of vasopressors are predictors that already were associated with higher mortality for AKI or COVID patients ${ }^{36-40}$.

The presence of comorbidities as diabetes and hypetension indicates the importance of pre-existing conditions to the severity of COVID-19 and AKI. Even though hypertension is age-dependent factors, they remained as an independent risk factor in the final model. 
In AKI COVID patients, HA-AKI, sepsis as a cause of AKI or MODS attributables to SARS-CoV-2 infection, higher creatinine levels during hospitalization and use of KRT were related to worse outcome. We already know from the non-COVID-19 literature that HA-AKI and its severity are associated with worse clinical outcomes when compared to CA-AKI, for several reasons. Seeing that ICU patients are in severe condition due to increased sepsis- associated AKI, increased aged and comorbidities, more affected by multiorgan failure leading to metabolic and fluid demands higher than renal capacity and need KRT, the unfavourable outcome can be justified ${ }^{41}$.

In addition to the adverse mechanical effect on lung function (decrease in forced expiratory volume and forced vital capacity), it has been proposed that the metabolic alterations produced by COVID-19 could decrease cardiorespiratory reserves in the face of a stressor, enhance dysregulation of the immune system, and favor a prothrombotic and proinflammatory state, all of which are physiopathological phenomena relevant in SARS-CoV-2 infection ${ }^{40-42}$..

Our study showed that the shorter time from COVID-19 symptoms to hospitalization and to AKI developmnet was associated with more disease severity and death. Similar results were observed by de Rodríguez-Molinero et al and this suggests that a longer presentation may be a reflection of less aggressive disease, which is an interesting observation ${ }^{42}$.

Some predictive models of COVID-19 had a better accuracy but combine data from radiology, biochemistry, and vital signs, which can also limit its application in a remote assessment. In a review of COVID-19 predictive models, the majority were low-quality models because did not include a description of the study population, and calibration of the model predictions was rarely assessed. Without a validation cohort, their reported performance is probably optimistic limiting the conclusion and applications ${ }^{43}$. Differently, we adopted a machine learning approach and we confirm the accuracy with a validation cohort. We also used well describe cohort data to fit the model and followed TRIPOD recommendations ${ }^{21}$.

This predictive model was developed with a large number of patients resulting from Latin America effort to analyze and report the risk for death in AKI COVID-19 patients.

Our study has a few limitations that need to be mentioned. First, we analyzed data from several countries from Latin America, in a period where clinical protocols for the disease were still being established, so this could afect the incidence of prognostic outcomes and may not directly generalize to other periods. Another one, it was an analysis subject to the drawbacks of patient records review. Obesity was not directly measured by body mass index, but rather clinical defined, gathered from medical records, which may have led to underreporting. Due to the pragmatic study design, laboratory exams were performed at the discretion of the treating physician, and we did not have a full dataset on all laboratory parameters of interest available. Some laboratory parameters, which proved to be of prognostic relevance in other studies, were not available for at least $2 / 3$ of patients in our sample. Therefore, we cannot rule out that 
variables with a higher proportion of missing data would have had a significant impact on mortality prediction.

\section{Strengths And Limitations}

A major strength of the AKI-COV scoring is its simplicity, the use of objective parameters, what helps to reduce inter-user variability, easily available at the emergency department presentation, even in underresourced settings. A major strength of this study is that it followed strict methodological criteria, recommended by TRIPOD checklist and PROBAST [43], and was based on robust sample of patients with laboratory confirmed SARS-CoV-2 infection, from a collaboration among researchers from 12 countries and 57 cities, including public, private and mixed hospitals of different sizes, to ensure diversity of the population studied and representativeness of the intended target population.

AKI COV score may help clinicians to make a prompt and reasonable decision to optimize AKI COVID 19 patient management and potentially reduce mortality. However, further prospective studies are needed to investigate whether the use of the score in the emergency department or ICU indeed trigger actions that result in reduced complications and hospital mortality.

\section{Conclusion}

In conclusion, we developed and validated the AKIV COV score and a web-based risk calculator. This score, based on age, comorbidity, leukocytos number, ALT level, need mechanical ventilation and vasopressor at admission, time from COVID symptons to hospitalziation and to AKI and AKI characteristics is an inexpensive tool, showed to objectively and accurately predict in-hospital mortality in AKI COVID-19 patients. It may be used at bedside for earlier identification of in-hospital mortality risk and, thus, inform clinical decisions and the assignment to the appropriate level of care and treatment for AKI COVID-19 patients

\section{Declarations}

\section{Latin American AKI COVID-19 Registry investigators (in alphabetical order):}

Adriana Flores (Mexico City, Mexico), Alejandro Ferreiro (Montevideo, Uruguay), Ana Paula Villa (Guadalajara, Mexico), Aureliano Ferrari (Asuncion, Paraguay), Benedito Pereira (Sao Paulo, Brazil), Caio Costa (Petropolis, Brazil), Carlos F. Varela (Buenos Aires, Argentina), Caroline de Acevedo (Rio de Janeiro, Brazil), Cristina Carlino (Rosario, Argentina), Daniela Ponce (Botucatu, Brazil), Diego Janiques (Petrópolis, Brazil), Emmanuel Burdmann (Sao Paulo,Brazil), Ereic Roessler (Santiago de Chile, Chile), Giannyigg (ICA, Peru), Giovanni Arrivillaga (Quetzaltenango, Guatemala), Gisselle Guzman (Santo Domingo, Dominican Republic), Galo Andrés Coronel (Buenos Aires, Argentina), Gustavo Aroca (Barranquilla, Colombia), Jonathan Chavez (Guadalajara, Mexico), Jose Ubillo (Mexico City, Mexico), Julieta Raño (Buenos Aires, Argentina), Lilia Rizo-Topete (Monterrey, Mexico), Luis Rodríguez (Tucumán, Argentina), Luis Yu (Sao 
Paulo, Brasil), Marcos Colombo (Jau, Brazil), Mariana B. Pereira (Sao Paulo, Brazil), Mauricio YounesIbrahim (Rio de Janeiro, Brazil), Natalia Rivas (Rojas, Argentina), Nelson Rojas (Buenos Aires, Argentina), Roger Ayala (Asuncion, Paraguay), Raul Ivan (Bolivia), Raúl Lombardi (Montevideo, Uruguay), Rolando Claure-Del Granado (Cochabamba, Bolivia), Serena Amor (Montevideo, Uruguay), Washington Osorio (Quito, Ecuador), Yanissa Venegas (Lima, Peru), Guillermo Rosa-Diez (Buenos Aires, Argentina)

\section{Acknowlegments}

We would like to thank the hospitals that were part of this 57 collaboration in 12 countries, for supporting this project:

We also thank all the clinical staff at those hospitals, which cared for the patients.

I would like the names of the individual members of the Group to be searchable through their individual PubMed record

\section{Funding}

This study was partially funded by Latin American Society of Nephrology and Hypertension (SLANH)

\section{Role of the funder/sponsor}

The sponsors had no role in design and conduct of the study; collection, management, analysis, and interpretation of the data; preparation, review, or approval of the manuscript; and decision to submit the manuscript for publication.

\section{Conflicts of interest}

The authors declared no potential conflicts of interest with respect to the research, authorship, and/or publication of this article.

\section{Data availability statement}

Data are available upon reasonable request.

\section{Transparency declaration}

The lead authors (DP and RL) affirm that the manuscript is an honest, accurate, and transparent account of the study being reported; that no important aspects of the study have been omitted; and that any discrepancies from the study as originally planned (and, if relevant, registered) have been explained.

\section{Ethical Approval}

Not aplicable (register study) 
Not aplicable (register study)

\section{Consent for publication}

All authors declare to aprove the paper for publication

\section{Author contribution}

Substantial contributions to the conception or design of the work: DP, LGMA

Substantial contributions to the acquisition, analysis, or interpretation of data for the work: RL, RCR, AF

Drafted the work: DP, LGMA

Revised the manuscript critically for important intellectual content: all authors.

Final approval of the version to be published: all authors.

Agreement to be accountable for all aspects of the work in ensuring that questions related

to the accuracy or integrity of any part of the work are appropriately investigated and resolved: DP and LGMA.

\section{References}

1. Cimerman S, Chebabo A, Cunha CAD, Rodriguez-Morales AJ. Deep impact of COVID-19 in the healthcare of Latin America: the case of Brazil. Braz J Infect Dis. 2020;24(2):93-5.

2. Barberia LG, Gomez EJ. Political and institutional perils of Brazil's COVID-19 crisis. Lancet. 2020;396(10248):367-8.

3. World-o-meters.info in https://www.worldometers.info/coronavirus/Covid.

4. - Yang Q, Yang X. Incidence and risk factors of kidney impairment on patients with COVID-19: A metaanalysis of 10180 patients. PLoS ONE. 2020;15(11):e0241953. https://doi.org/10.1371/journal.pone.0241953.

5. - Li Z, Wu M, Yao J, Guo J, et al. Caution on Kidney Dysfunctions of COVID-19 Patients. SSRN Electron J. 2020:1-25. doi:10.2139/ssrn.3559601.

6. - Hirsch JS, Ng JH, Ross DW, et al. Acute kidney injury in patients hospitalized with COVID-19. Kid Int. 2020; 98: 209-18. doi:10.1016/ j.kint.2020.05.006.

7. - Diao $B$, Wang $C$, Wang $R$, et al. Human kidney is a target for novel severe acute respiratory syndrome coronavirus 2 (SARS-CoV-2) infection. medRxiv. 2020;2:1-17. doi:10.1101/2020.03.04.20031120.

8. Kudose S, Batal I, Santoriello D, et al. Kidney biopsy findings in patients with COVID-19. J Am Soc Nephrol. 2020;31:1959-68. doi:10.1681/ASN.2020060802. 
9. Cheng Y, Luo R, Wang K, et al. Kidney impairment is associated with inhospital death of COVID-19 patients. Nephrology. 2020 Feb. https://doi.org/10.1101/2020.02.18.20023242.

10. Guan WJ, Ni ZY, Hu Y, et al. Clinical characteristics of coronavirus disease 2019 in China. N Engl J Med. 2020;382:1708-20.

11. Aggarwal S, Garcia-Telles N, Aggarwal G, Lavie C, Lippi G, Henry BM. Clinical features, laboratory characteristics, and outcomes of patients hospitalized with coronavirus disease 2019 (COVID-19): Early report from the United States. Diagnosis. 2020; 7: 91-96. https://doi.org/10.1515/dx-20200046 PMID: 32352401.

12. Hirsch JS, Ng JH, Ross DW, et al. Acute kidney injury in patients hospitalized with COVID-19. Kidney Int. 2020;98:209-18.

13. Zamoner W, Santos CAS, Magalhaes LE, et al. Acute Kidney Injury in COVID-19: 90 Days of the Pandemic in a Brazilian Public Hospital. Front Med. 2021;8:622577. doi:10.3389/fmed.2021.622577.

14. Mahase E. Covid-19: increasing demand for dialysis sparks fears of supply shortage. BMJ. 2020;369:m1588.

15. Liang W, Liang H, Ou L, et al. Development and Validation of a Clinical Risk Score to Predict the Occurrence of Critical Illness in Hospitalized Patients With COVID-19. JAMA Intern Med. 2020;180:1081-9.

16. Burdick H, Lam C, Mataraso S, et al. Prediction of respiratory decompensation in Covid-19 patients using machine learning: The READY trial. Comput Biol Med. 2020;124::103949.

17. Magro B, Zuccaro V, Novelli L, et al. Predicting in-hospital mortality from Coronavirus Disease 2019: A simple validated app for clinical use. PLoS One 2021; 16:e0245281 (2021).

18. Greenhalgh T, Koh GCH. C. J. Covid-19: a remote assessment in primary care. BMJ. 2020;25:368: $\mathrm{m} 1182$.

19. Latin America COVID-AKI. Registy: an observational, prospective, longitudinal and multinational study (submitted) and presented in WCN 2020.

20. Cerda J, Mohan S, Garcia-Garcia G, et al. Acute Kidney Injury Recognition in Low and Middle Income Countries. Kid Int Rep. 2017;2(4):530-43.

21. Moons KGM, Altman DG, Reitsma JB, et al. Transparent reporting of a multivariable prediction model for individual prognosis or diagnosis (TRIPOD): Explanation and elaboration. Ann Intern Med. 2015;162(1):W1-73.

22. Chawla NV, Bowyer KW, Kegelmeyer HL. W.P. SMOTE: synthetic minority over-sampling technique. J Artif Intell Res. 2002;16:321-57.

23. K R. Use of Brier score to assess binary predictions. J Clin Epidemiol. 2010;63:938-9.

24. Rodríguez-Pérez R. B. J. Interpretation of machine learning models using shapley values: application to compound potency and multi-target activity predictions. J Comput Aided Mol Des. 2020;34:101326. 
25. Yan L, Zhang HT, Goncalves J, et al. An interpretable mortality prediction model for COVID-19 patients. Nat Mach Intell. 2020;2:283-8.

26. Batista AFM, Miraglia JL, Donato HR, Chiavegatto Filho ADP. COVID-19 diagnosis prediction in emergency care patients: A machine learning approach. medRxiv. 2020. https://doi.org/10.1101/2020.04.04.20052092.

27. Heldt FS, Vizcaychipi MP, Peacock S, et al. Early risk assessment for COVID-19 patients from emergency department data using machine learning. medRxiv. 2020. https://doi.org/10.1101/2020.05.19.20086488.

28. Gao Y, Cai GY, Fang W, et al. Machine learning based early warning system enables accurate mortality risk prediction for COVID-19. Nat Commun. 2020;11:5033.

29. Cheng FY, Joshi $H$, Tandon $P$, et al. Using machine learning to predict ICU transfer in hospitalized COVID-19 patients. J Clin Med 2020; 9(6). https:// doi.org/10.3390/jcm9061668.

30. Cao X. COVID-19: Immunopathology and its implications for therapy. Nat. Rev. Immunol. Internet. 2020; 20, 269-70. doi. org/10.1038/s41577-020-0308-3 30. Rath, D. et al. Impaired cardiac function is associated with mortality in patients with acute COVID-19 infection. Clin. Res. Cardiol. 2020. https://doi.org/10.1007/s00392-020-01683-0.

31. Bajwa E, Khan UA, Januzzi JL, et al. Plasma C-reactive protein levels are associated with improved outcome in ARDS. Chest. 2009;136(2):471-80.

32. Chen W, Zheng KI, Liu S, et al. Plasma CRP level is positively associated with the severity of COVID19. Ann Clin Microbiol Antimicrob. 2020;19:18.

33. Wang G, Wu C, Zhang Q, et al. C-Reactive protein level may predict the risk of COVID-19 aggravation. Open Forum Infect Dis. 2020. https://doi. org/10.1093/ofd/ofaa153.

34. Fuentes E, Fuentes M, Alarcon M, Palomo I. Immune system dysfunction in the elderly. An Acad Bras Cienc. 2017;89(1):285-99.

35. Sherwani S, Khan MWA. Cytokine Response in SARS-CoV-2 Infection in the Elderly. Journal of Inflammation Research. 2020;13:737-47.

36. Yadav R, Aggarwal S, Singh A. SARS-CoV-2-host dynamics: increased risk of adverse outcomes of COVID-19 in obesity. Diabetes Metab Syndr. 2020;14:1355-60. doi:10.1016/j.dsx.2020.07.030.

37. Sanchis-Gomar F, Lavie CJ, Mehra MR, Henry BM, Lippi G. Obesity and outcomes in COVID-19: when an epidemic and pandemic collide. Mayo Clin Proc. 2020); 95:1445-53. doi:

10.1016/j.mayocp.2020.05.006.

38. Bucuvic EM, Ponce D, Balbi AL. Fatores de risco para mortalidade na lesão renal aguda. Rev Assoc Med Bras. 2011;57:158-63. doi:10.1590/S0104-42302011000200012.

39. Garcia TPR, RomeroMP, Poletti NA, Cesarino CB, Ribeiro RCHM. Principais motivos de internação do paciente com insuficiência renal aguda na unidade de terapia intensiva. Arq Ciênc Saúde. $2005 ; 12: 146-50$. 
40. Santos ER, Matos JD. Perfil epidemiologico dos pacientes com injúria renal aguda em uma unidade de terapia intensiva. Arq Cat deMed. 2008;37:1806-20.

41. Ostermann $M$, Joannidis $M$, Pani $A$, et al. 17th acute disease quality initiative (ADQI) consensus group patient selection and timing of continuous renal replacement therapy. Blood Purif. 2016;42(3):224-37. doi:10.1159/0004485066.

42. Rodríguez-Molinero A, Gálvez-Barrón C, Miñarro A, et al. on behalf of the COVID-19 Research Group of CSAPG. Association between COVID-19 prognosis and disease presentation, comorbidities and chronic treatment of hospitalized patients https://doi.org/10.1371/journal.pone.0239571.

43. Liang W, Liang H, Ou L, et al. Development and validation of a clinical risk score to predict the occurrence of critical illness in hospitalized patients with COVID-19. JAMA Internal Medicine. 2020;180(8):1081-9.

\section{Figures}




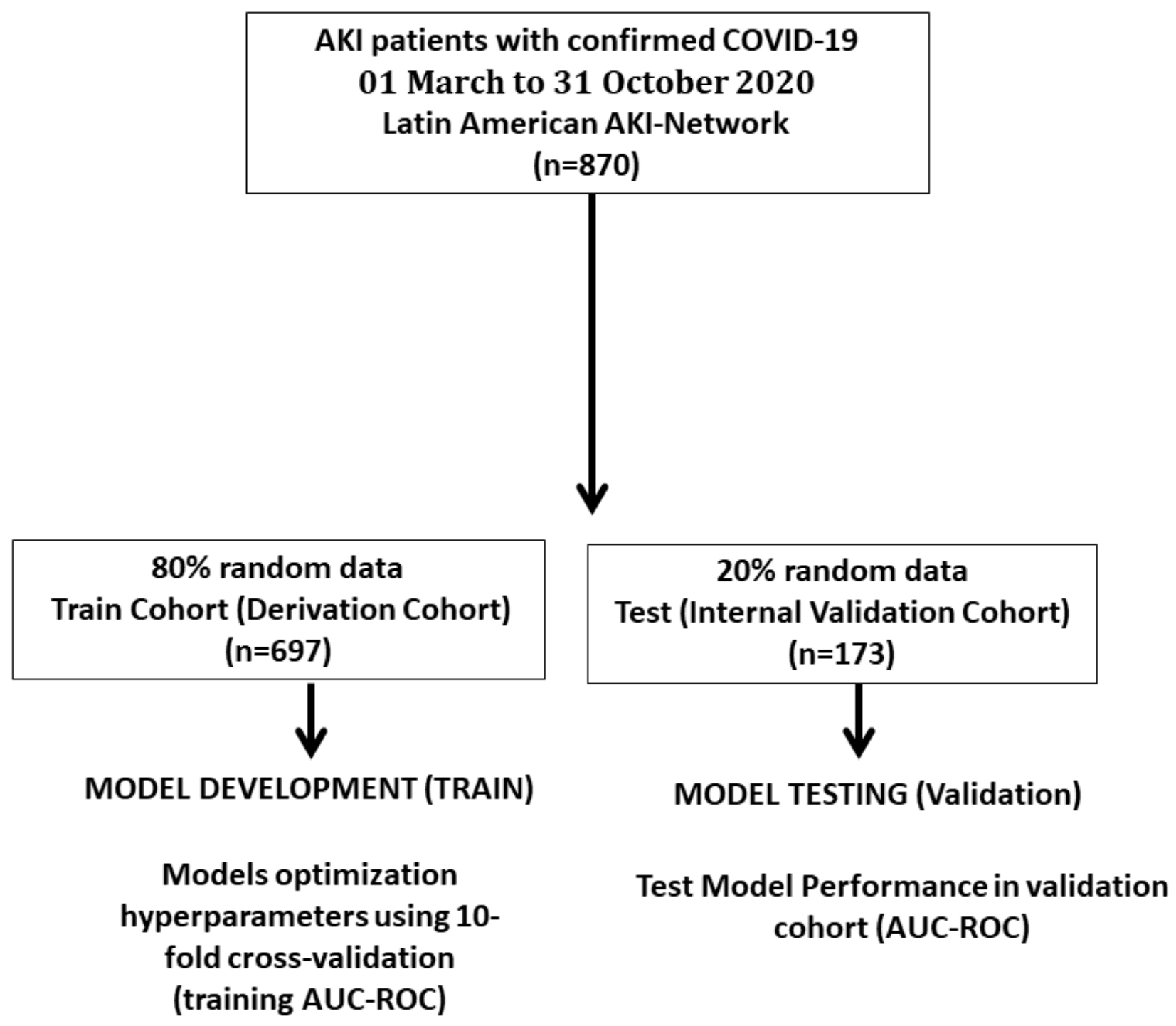

RT-PCR: reverse-transcription polymerase chain reaction

AUC-ROC: area under the receiver operating characteristic curve

Figure 1

Derivation cohort or train set and the internal validation cohort or test set 


\section{Receiver Operator Characteristic}

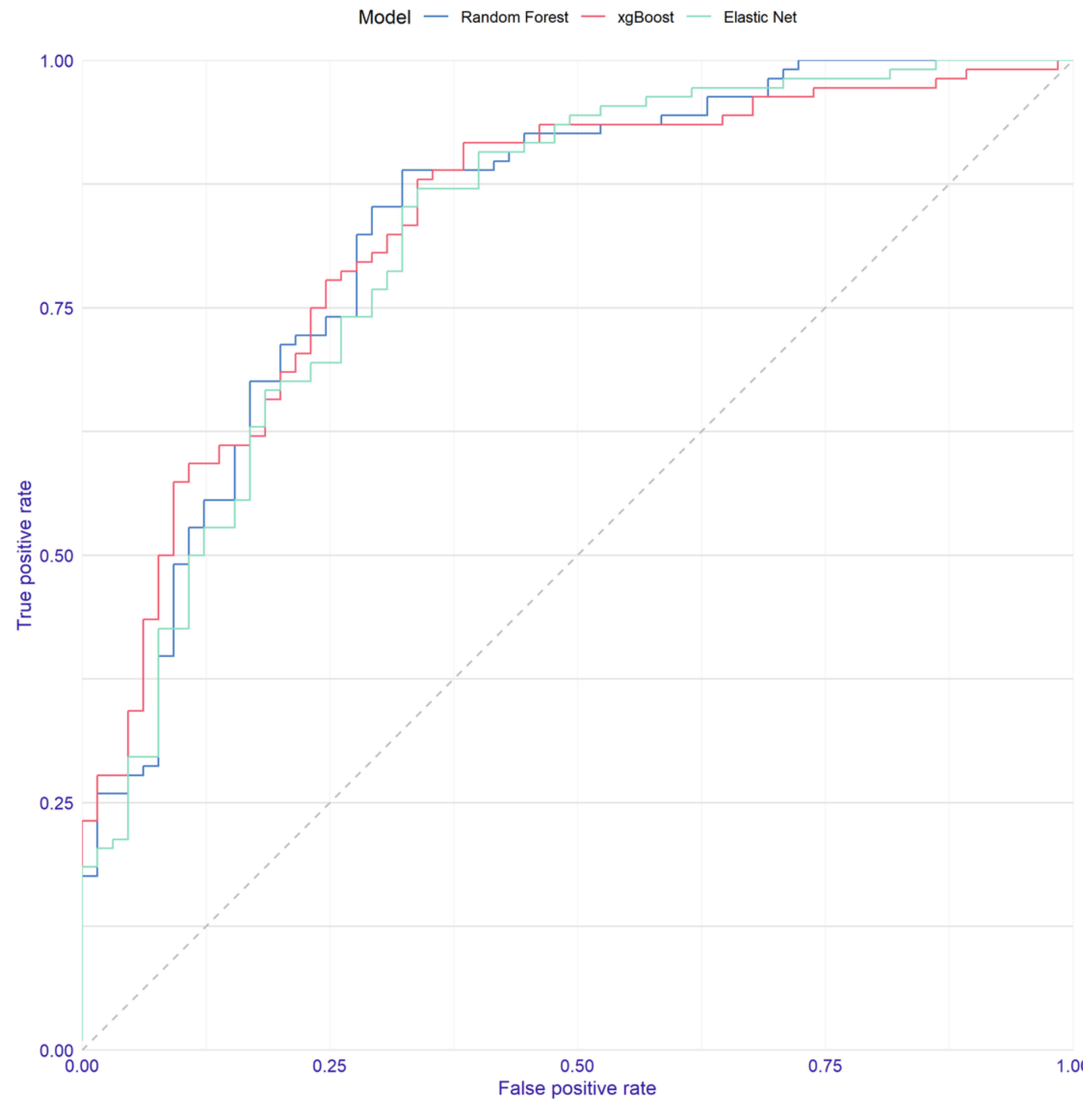

\section{Figure 2}

AUC-ROC in the derivation cohort of AKI COVID-19 mortality predictive models 


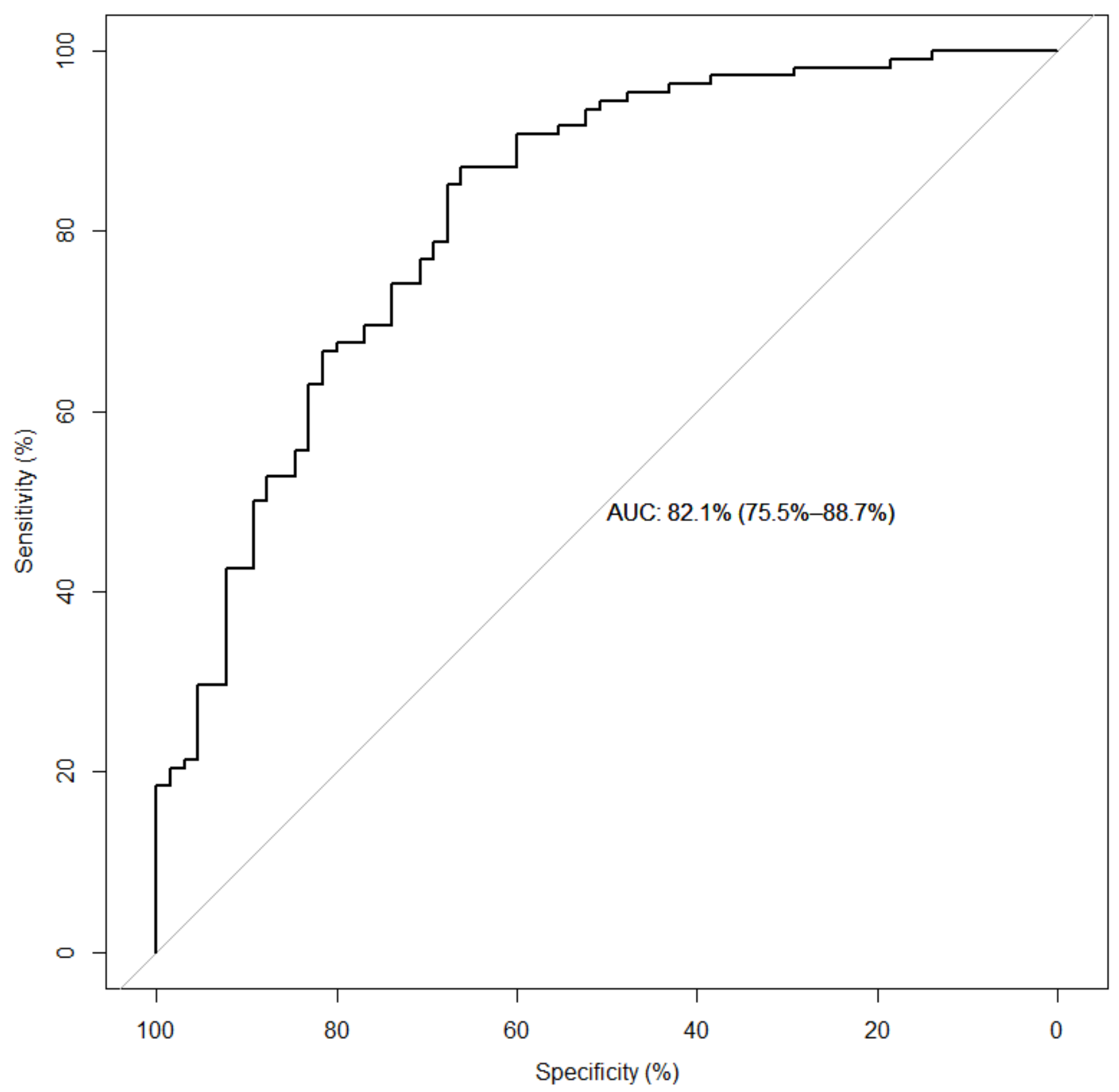

Figure 3

Confusion Matrix of AKI COVID-19 in hospital mortality in derivation cohort (test set) 


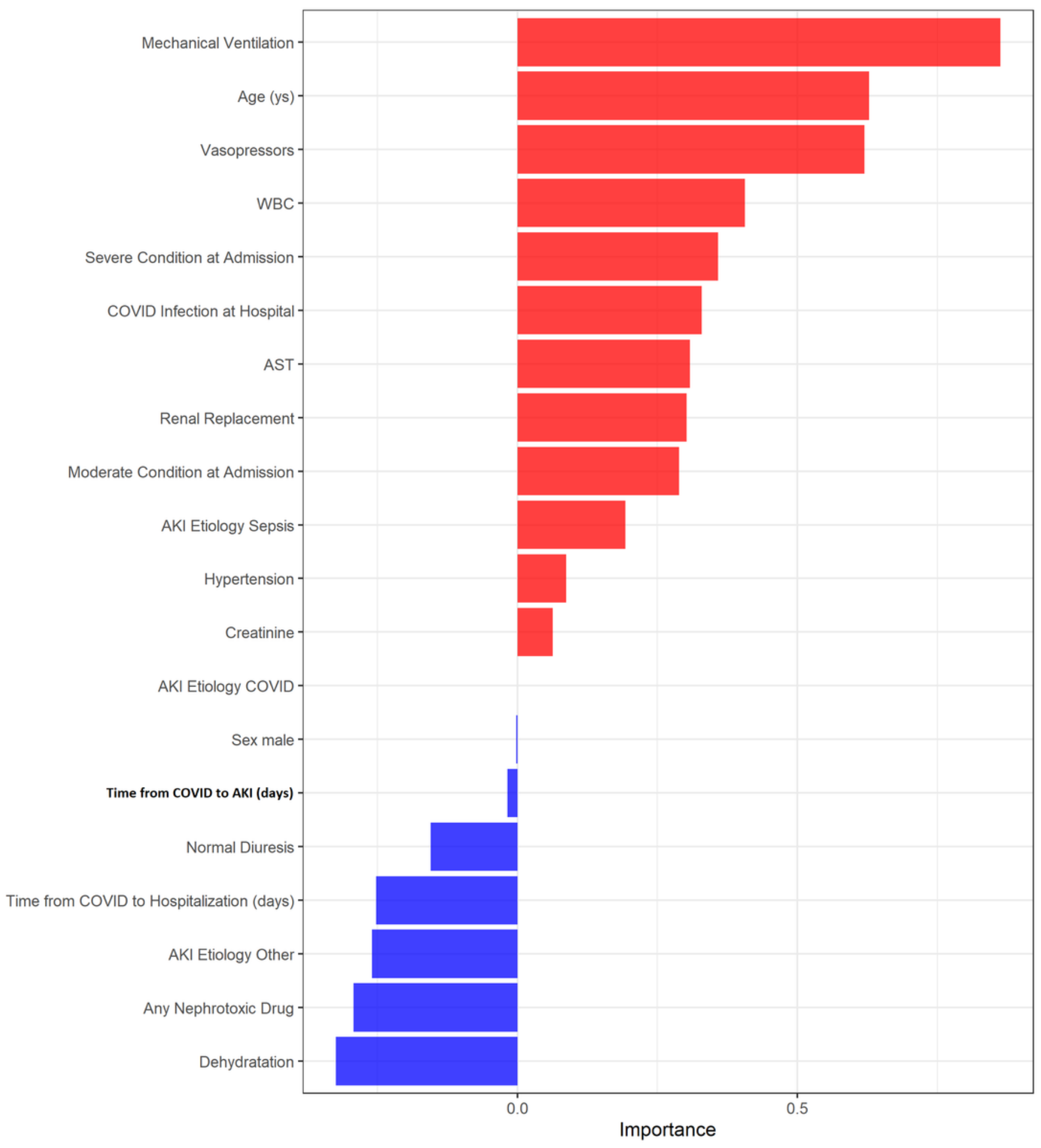

\section{Figure 4}

Coefficients of Elastic Net of AKI COVID-19 in-hospital mortality model (Variable Importance). The red bars represent the variables related with the probability of death, whereas the blue bars were related with the probability of surviving. The model was fitted with 15 predictors and we derived natural splines in the variables age and eGFR. The natural splines computed a different risk for each stratum aiming to capture the non-linear association between these predictors and outcome. 
Patient 01

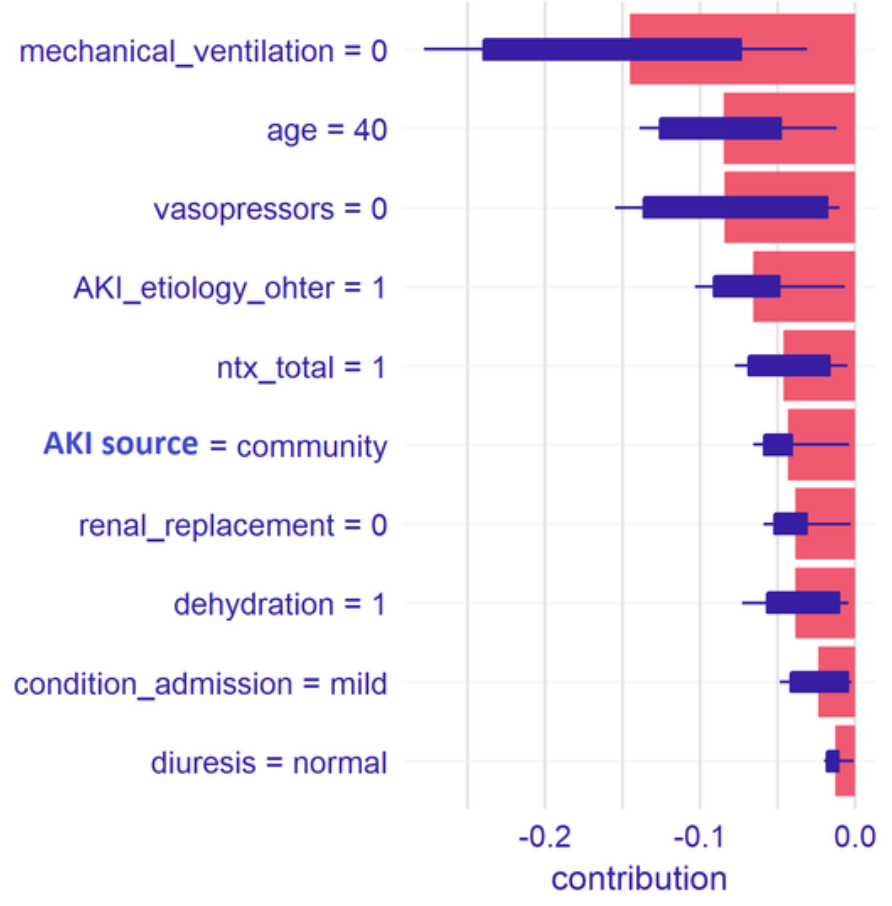

\section{Patient 03}

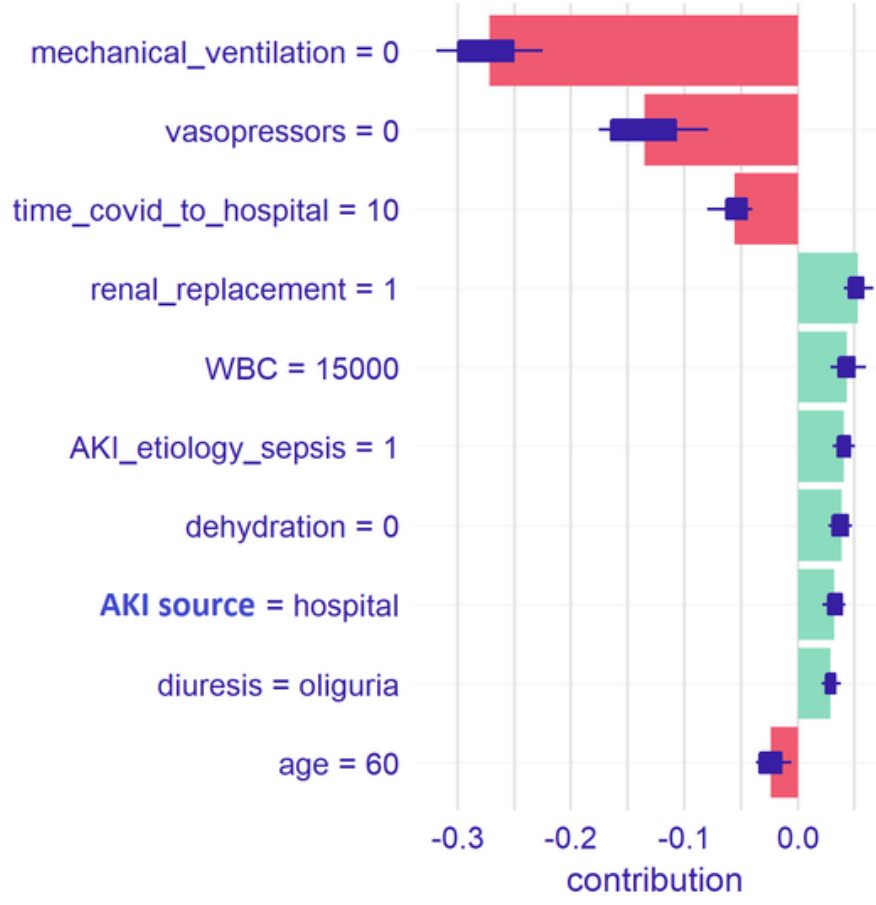

Patient 02

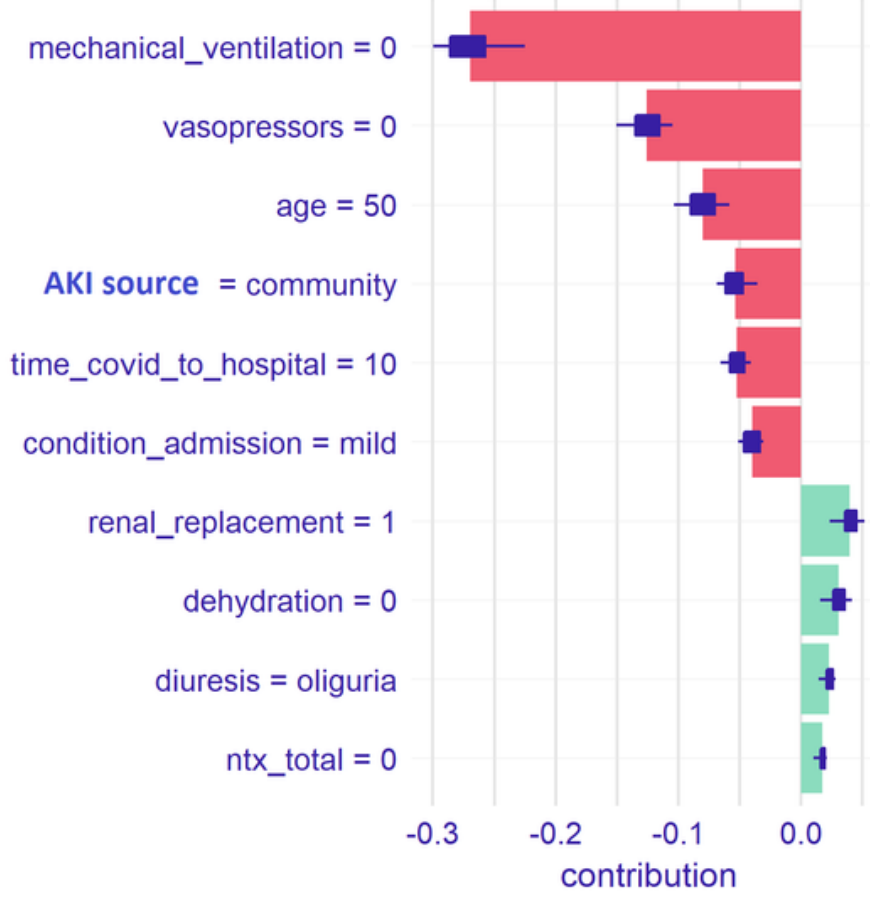

\section{Patient 04}

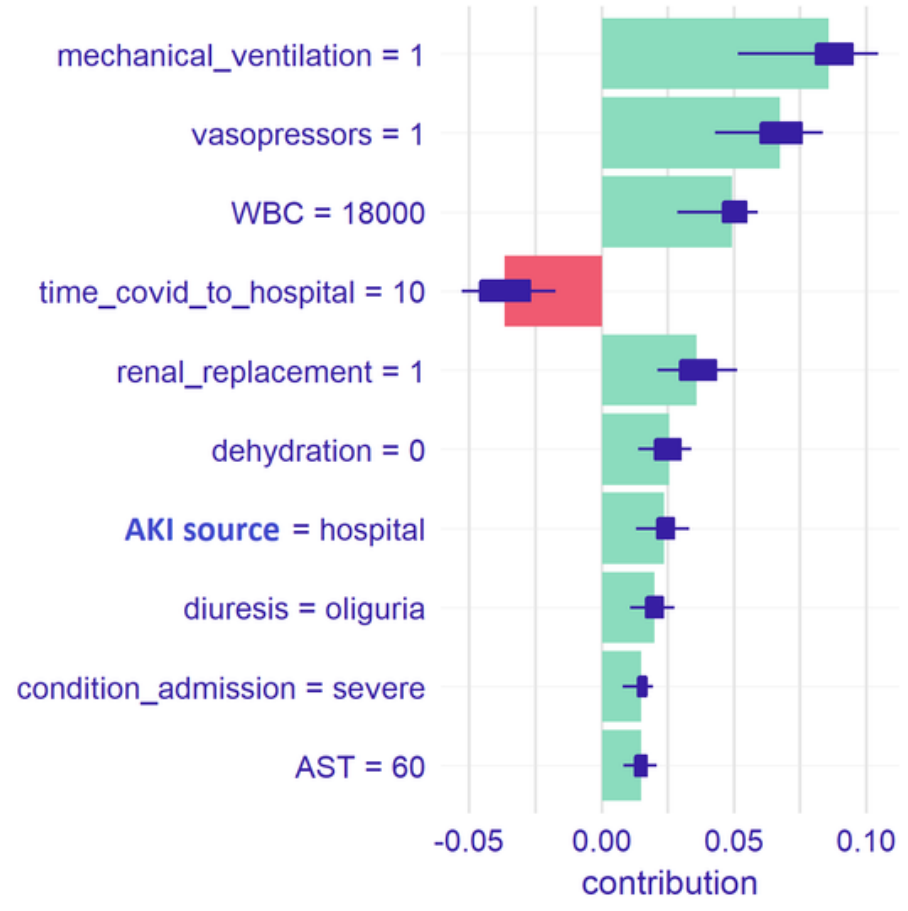

\section{Figure 5}

Shapley Additive Explanations (SHAP plot) showed the contribution of each predictor in AKI COVID-19 inhospital mortality score. 
870 AKI patients with confirmed COVID-19

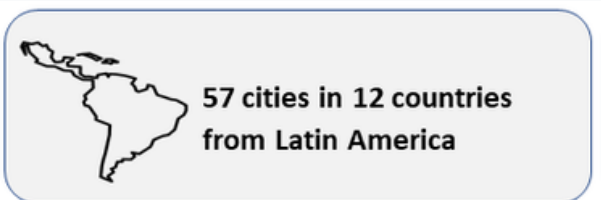

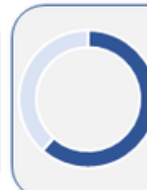

$62.5 \%$ progressed to death Outcome: Death in-hospital

\section{AKI-COV score: COVID-19 risk stratification among AKI patients}

Predictors Avaiable

\begin{tabular}{|ll|}
\hline Demographic: & Age; male sex \\
Comorbidities: & Hypertesion; Dehydratation \\
\hline Codition at Admission: & Mild; Moderate; Severe \\
\hline AKI Etiology: & AST; WBC; creatinine \\
Condition: & COVID; Sepsis; Other \\
\hline
\end{tabular}

\section{Score-Based Prediction}

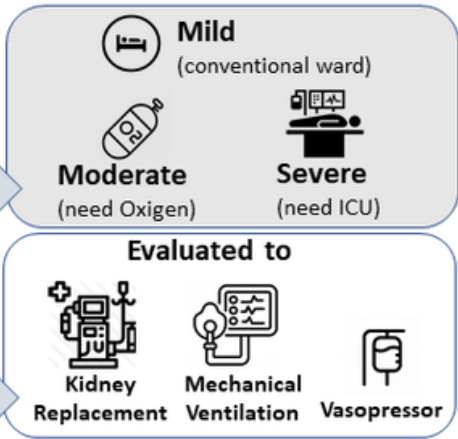

The model may assist in identifying hospitalized AKI COVID-19 patients that may require more intensive monitoring

MODEL $\quad \begin{gathered}\text { Probability } \\ \text { Death }\end{gathered}$

\section{Figure 6}

Visual abstract 